\title{
Caracterização de Polietilenos Lineares de Baixa Densidade I. Determinação do Teor de Ramificação por Métodos Espectroscópicos
}

\author{
Antonio C. Quental, Lilian S. Hanamoto, Maria I. Felisberti \\ Instituto de Química, UNICAMP
}

Resumo: Os polietilenos lineares de baixa densidade (LLDPE) apresentam propriedades variando amplamente em função da concentração e do tipo de comonômero utilizado na copolimerização, além do tipo de catalisador. Neste trabalho diversos tipos de LLDPE obtidos com diferentes tipos de comonômeros e catalisadores foram caracterizados estruturalmente por RMN $-{ }^{13} \mathrm{C}$ e FTIR. Os resultados obtidos por meio destas duas técnicas foram discutidos e comparados.

Palavras-chave: Polietileno, caracterização, $R M N^{13} \mathrm{C}, \mathrm{FTIR}$, comonômeros.

\section{Linear Low Density Polyethylene Characterization - I. Spectroscopic Methods for Comonomer Content Determination}

Abstract: Linear Low Density Polyethylenes (LLDPE) show a broad variation in their properties by changing the type and comonomer content, besides the catalyst used. LLDPE properties change depending on the comonomer and its concentration at polymer chains and also by the catalyst used in polymerization. Several types of LLDPE containing different comonomers in various concentrations, obtained by metallocenic and Ziegler-Natta catalyst, were characterized by ${ }^{13} \mathrm{C}$ NMR and FTIR. The results obtained from these two techniques were discussed and compared.

Keywords: Polyethylene, characterization, ${ }^{13}$ C NMR, FTIR, comonomer.

\section{Introdução}

O polietileno linear de baixa densidade (LLDPE), apresenta uma arquitetura molecular intermediária à do polietileno de alta densidade (HDPE) e a do polietileno de baixa densidade (LDPE). O LLDPE é um polímero linear com teores variados de ramificações de comprimento constante. Ambos, LLDPE e o LDPE possuem ramificações de cadeia curta, sendo a origem destas totalmente diferentes. No LDPE, as ramificações resultam do processo de polimerização via radicais livres, através de reações de transferência de hidrogênio. No LLDPE as ramificações são introduzidas nas cadeias por meio da copolimerização de eteno com uma $\alpha$-olefina, utilizando-se catalisadores de metais de transição. Assim, as ramificações no LLDPE são curtas e constantes, ao contrário das ramificações nos LDPE, que podem ter diversos comprimentos, sendo algumas relativamente longas. $\mathrm{O}$ polietileno de alta densidade (HDPE) possui uma quantidade extremamente pequena de ramificações ${ }^{[1-3]}$.

Na produção das resinas comerciais de LLDPE, as $\alpha$-olefinas mais utilizadas são o propeno, o 1-buteno, o 1-hexeno e o 1-octeno, em concentrações na faixa de 2 a $15 \%$ mol. Também pode-se utilizar misturas das $\alpha$-olefinas, como por exemplo, eteno, propeno e 1-buteno, para pro- duzir um terpolímero com ramificações com dois comprimentos distintos. Na copolimerização, o grupo vinila do comonômero é incorporado à cadeia principal. A ramificação gerada possui, então, dois carbonos a menos que o monômero de partida. Assim, na copolimerização do propeno tem-se um carbono como ramificação; quando utiliza-se o 1-buteno a ramificação gerada possui dois carbonos e assim por diante ${ }^{[1,2,4]}$.

Os catalisadores utilizados na produção do LLDPE são do tipo Ziegler-Natta ou do tipo metalocênico. Dependendo do catalisador utilizado, o LLDPE pode apresentar diferentes microestruturas, em relação à massa molar e à sua distribuição, bem como ao teor e à distribuição dos comonômeros ao longo da cadeia polimérica. Isto leva os LLDPE a apresentarem diferentes características físicas e mecânicas entre $\mathrm{si}^{[1,2,5]}$.

Embora o controle do tipo e da concentração de comonômeros seja direto, o controle da distribuição das ramificações é mais alusivo. A distribuição das ramificações, considerada um fator importante na resistência dos filmes de LLDPE, está relacionada com as condições de processo e também com o tipo de catalisador utilizado. Atualmente, existem os LLDPE, que além das ramificações de cadeias curtas (RCC), possuem também pequenas quantidades controladas de ramificações de cadeias longas (RCL) ${ }^{[1,2,6-8]}$.

Autor para correspondência: Maria Isabel Felisberti, Grupo de Pesquisa em Polímeros (GPPol), Instituto de Química, UNICAMP, Caixa Postal 6154, CEP:13083-970, Campinas, SP. E-mail: misabel@iqm.unicamp.br 
O desenvolvimento de catalisadores heterogêneos de Ziegler-Natta durante os últimos 40 anos é, sem dúvida, o principal responsável pelo crescimento do volume produzido e utilizado de plásticos poliolefínicos. Este tipo de catalisador possui um centro metálico ativo que pode ocupar uma grande variedade de sítios na estrutura cristalina do catalisador. Deste modo tem-se sítios catalíticos com diferentes reatividades ("multisítio"). Como conseqüência os produtos obtidos por este tipo de catalisador apresentam ampla distribuição de massa molar e de outras características moleculares, como o teor de comonômero e a sua taticidade, comparativamente aos produtos obtidos por catalisadores de único sítio. A pesquisa sobre os catalisadores Ziegler-Natta tem objetivado principalmente a redução desses multisítios e a mistura desses catalisadores com outros. Os catalisadores coordenados metalocênicos baseados em compostos ciclopentadiênicos de metais de transição como titânio, zircônio e háfnio, polimerizam eficientemente eteno e propeno, além de outras $\alpha$-olefinas lineares e cíclicas. Esses sistemas são capazes de polimerizar também o estireno e monômeros funcionalizados como o cloreto de vinila, a acrilonitrila e outros. Em contraste com os catalisadores Ziegler-Natta, os metalocênicos possuem uma única espécie ativa com um tipo de coordenação bem definido (único sítio), que possibilita a obtenção de resinas com distribuição de massa molar estreita e alto teor de comonômeros incorporado e distribuídos homogeneamente nas cadeias do polímero. Além disso, a tecnologia Insite, desenvolvida pela Dow Chemical, utiliza um catalisador de geometria tensionada que pode criar mistura controlada de ramificações de cadeias curtas e longas na cadeia principal.

Os métodos para a determinação do teor de ramificações estão muito bem estabelecidos na literatura destacando-se a ressonância magnética nuclear de ${ }^{13} \mathrm{C}$ e a espectroscopia no infravermelho. Para ambas as técnicas existem normas técnicas que estabelecem os procedimentos experimentais e o tratamento de dados. O objetivo deste trabalho é apresentar de uma forma mais clara a aplicação destas normas na determinação do teor de comonômeros em LLDPE com características microestruturais distintas. Os resultados obtidos pela aplicação das duas metodologias são comparados ${ }^{[1,2,6-10]}$.

\section{Experimental}

\section{Materiais Utilizados}

A Tabela 1 mostra a relação dos polietilenos lineares utilizados, bem como as suas características químicas e físicas. Os polietilenos PL 1880, Dowlex 2045 e Elite 5400 foram produzidos pela Dow Chemical e o LFH 118 pela Braskem S.A.. O PL 1880 pertence à família de LLDPE conhecida como

Tabela 1. Características físico-químicas dos LLDPE utilizados.

\begin{tabular}{|c|c|c|c|c|}
\hline & PL 1880 & Dowlex 2045 & Elite 5400 & LFH 118 \\
\hline Catalisador & Metalocênico & Ziegler-Natta & $\begin{array}{l}\text { Ziegler-Natta / } \\
\text { Metalocênico }\end{array}$ & Ziegler-Natta \\
\hline Comonômero & 1-octeno & 1-octeno & 1-octeno & 1-hexeno \\
\hline Índice de Fluidez (g/10 min) ${ }^{a}$ & 1 & 1 & 1 & 1 \\
\hline $\bar{M}_{n} \times 10^{3}(\mathrm{~g} / \mathrm{mol})^{b}$ & 76 & 44 & 58 & 46 \\
\hline $\bar{M}_{w} \times 10^{3}(\mathrm{~g} / \mathrm{mol})^{b}$ & 172 & 228 & 220 & 223 \\
\hline$\overline{\mathbf{M}}_{\mathbf{w}} / \overline{\mathbf{M}}_{\mathrm{n}}$ & 2,3 & 5,2 & 3,7 & 5 \\
\hline $\mathbf{T}_{\mathrm{c}}\left({ }^{\circ} \mathbf{C}\right)^{\mathrm{c}}$ & 78 & 99 & 99 & 105 \\
\hline $\mathbf{T}_{\mathrm{f}}\left({ }^{\circ} \mathbf{C}\right)^{\mathrm{c}}$ & 101 & 123 & 124 & 126 \\
\hline$\chi(\%)^{\mathrm{c}}$ & 31 & 46 & 41 & 47 \\
\hline $\mathrm{d}\left(\mathrm{g} / \mathrm{cm}^{3}\right)^{\mathrm{a}}$ & 0.902 & 0.920 & 0.916 & 0.920 \\
\hline Ramificação & hexila e longas & hexila & hexila & butila \\
\hline $\mathrm{CH}_{3} / 1000 \mathrm{C}^{\mathrm{d}}$ & 34 & 11 & 13 & 14 \\
\hline Nomenclatura $^{\mathrm{e}}$ & Oct-M & Oct-ZN & Oct-ZN/M & Hex-ZN \\
\hline
\end{tabular}

(a) dado fornecido pelo fabricante

(b) obtido por GPC em 1,2,4-triclorobenzeno a $140{ }^{\circ} \mathrm{C}$.

(c) obtido por DSC

(d) obtido por $\mathrm{RMN}^{13} \mathrm{C}$.

(e) Nomenclatura utilizada ao longo deste trabalho. 
Afinnity, cuja característica principal é apresentar uma mistura controlada de ramificações curtas e longas na cadeia principal. O Elite 5400 é produzido através da mistura dos catalisadores metalocênicos e Ziegler-Natta, por último tem-se os LLDPE Dowlex e LFH 118 que diferem somente no tipo de comonômero e, portanto, na ramificação. A denominação adotada para os polietilenos neste trabalho indica o tipo de monômero (Oct $=1$-octeno; Hex $=1$-hexeno $)$ e o catalisador utilizado na polimerização ( $\mathrm{M}=$ metalocênico; $\mathrm{ZN}=$ ZieglerNatta). Assim os LLDPE PL 1880, Dowlex 2045; Elite 5400 e LFH 118 são designados Oct-M, Oct-ZN, Oct-ZN/M e Hex$\mathrm{ZN}$, respectivamente, conforme mostrado na Tabela 1.

\section{Ressonância Magnética Nuclear de Carbono ${ }^{13} \mathrm{C}\left(\mathrm{RMN}-{ }^{13} \mathrm{C}\right)$}

Os espectros de RMN foram obtidos em equipamentos VarianGemini 2000 e Brucker - 300AC/P, ambos operando a 75,4 $\mathrm{MHz}$ no modo de transformada de Fourier. Cada espectro foi obtido a $125^{\circ} \mathrm{C}$, com pulsos a $90^{\circ}$ e $15 \mathrm{~s}$ de tempo de espera (delay) entre os pulsos, com desacoplamento de prótons. As amostras em solução foram preparadas dissolvendo $0,4 \mathrm{~g}$ dos polímeros em $3 \mathrm{ml}$ de 1,2,4-triclorobenzeno com agitação a $130^{\circ} \mathrm{C}$. No instante de obter o espectro foi adicionado cerca de $1 \mathrm{ml}$ de benzeno deuterado.

\section{Espectroscopia no Infravermelho}

Os espectros de infravermelho foram obtidos em um equipamento Bomem MB Series Modelo B100. As amostras na forma de filmes foram obtidas prensando-se os polímeros a $180{ }^{\circ} \mathrm{C}$ por 2 minutos. Os espectros foram obtidos na faixa de 400 a $4000 \mathrm{~cm}^{-1}$ com uma resolução de $1 \mathrm{~cm}^{-1}$, com 32 acumulações.

\section{Resultados e Discussão}

\section{Ressonância Magnética Nuclear de Carbono 13}

A análise quantitativa em poliolefinas por meio da ressonância magnética nuclear de ${ }^{13} \mathrm{C}\left(\mathrm{RMN}^{13} \mathrm{C}\right)$ tem sido amplamente empregada devido à importância comercial destes materiais. Com esta técnica pode-se determinar o tipo, a distribuição e a quantidade de ramificações incorporadas na cadeia principal de uma poliolefina ${ }^{[1,2]}$.

A obtenção da quantidade de comonômero incorporada à cadeia do LLDPE foi realizada utilizando a norma ASTM D 5017-91 ${ }^{[11]}$. Esta norma foi submetida à ASTM por De Pooter e colaboradores ${ }^{[12]}$ e descreve como determinar a concentração de comonômero em porcentagem em mol e em porcentagem em massa, além do número de ramificações por 1000 átomos de carbonos $\left(\mathrm{CH}_{3} / 1000 \mathrm{C}\right)$.

A espectroscopia de $\mathrm{RMN}^{13} \mathrm{C}$ é um método absoluto que não requer padronização. No entanto, dois parâmetros devem ser bem definidos quando a análise quantitativa é realizada. O primeiro é o tempo de relaxação spin-rede $-\mathrm{T}_{1}$ e o segundo é o efeito NOE. O efeito NOE em polietilenos é constante para todos os carbonos do polímero. O tempo de espera (delay) entre cada pulso na análise por $\mathrm{RMN}^{13} \mathrm{C}$ deve ser no mínimo cinco vezes o tempo $T_{1}$ para que ocorra a relaxação de $99 \%$ dos carbonos. Para um tempo de espera menor que cinco vezes o $T_{1}$, haverá influência na intensidade dos sinais. Em uma ramificação, o grupo metila possui o maior $T_{1}, o$ qual aumenta proporcionalmente com o tamanho da ramificação, alcançando valores da ordem de 8 a $10 \mathrm{~s}$. Portanto, para uma análise quantitativa das ramificações em um polímero, um tempo de espera mínimo de 50 s seria necessário entre cada pulso. É fácil perceber, então, que seria necessário um tempo longo de análise, o que deixaria de ser uma análise prática ${ }^{[1,2,13-15]}$.

Bugada e Rudin ${ }^{[14]}$ utilizaram $\mathrm{RMN}^{13} \mathrm{C}$ para a quantificação das ramificações no polietileno de baixa densidade, chamando a atenção para um tempo longo de análise, associado à alta temperatura, que resulta na degradação do polímero.

Uma alternativa para viabilizar a análise é ignorar os carbonos com altos tempos de relaxação. Para os LLDPE a ramificação incorporada está ligada à cadeia principal através de um carbono terciário. Os tempos de relaxação dos carbonos de uma ramificação diminuem com a proximidade ao carbono terciário; entretanto aumentam na direção do grupo terminal, ou seja, do grupo $\mathrm{CH}_{3}$ que possui o maior tempo de relaxação de uma ramificação. Desta forma, os carbonos próximos ao carbono terciário possuem tempos de relaxação $\mathrm{T}_{1}$ menores que $2 \mathrm{~s}$. Assim, em uma análise um tempo de espera (delay time) de 10 a $15 \mathrm{~s}$ pode ser utilizado, sendo que os grupos metila são ignorados sem prejudicar a análise quantitativa $^{[13-15]}$

Os espectros de $\mathrm{RMN}^{13} \mathrm{C}$ foram obtidos a $125^{\circ} \mathrm{C}$, pois somente a altas temperaturas os polietilenos são solúveis devido à presença de uma fase cristalina. Altas temperaturas resultam na diminuição do tempo de relaxação dos polímeros e, por conseqüência, do tempo de análise. Além disso, quanto maior o tempo de análise, maior é o número de espectros acumulados, e mais resolvidos e intensos serão os picos obtidos, melhorando a análise quantitativa ${ }^{[1,2]}$.

A nomenclatura para designar os carbonos é a seguida por Usami e Takayama ${ }^{[15]}$. Os carbonos terciários são identificados como "br". Com letras gregas identifica-se os carbonos metilênicos da cadeia principal adjacentes aos carbonos terciários. Os carbonos das ramificações são identificados por $\mathrm{xBn}$, onde $\mathrm{n}$ é o número de carbonos na ramificação e $\mathrm{x}$ é a localização do carbono. Assim um carbono $1 \mathrm{~B}_{4}$ é o carbono da metila da ramificação butila, enquanto o $2 \mathrm{~B}_{6}$ é o

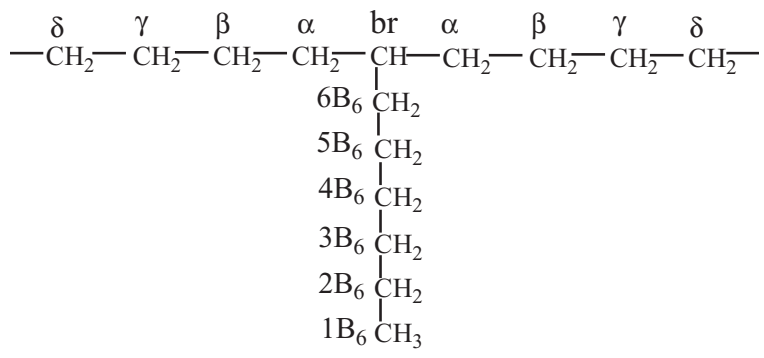

Figura 1. Nomenclatura utilizada para designar os carbonos nos espectros de $\mathrm{RMN}-{ }^{13} \mathrm{C}$. Exemplo relativo ao LLDPE obtido com o comonômero 1octeno. 

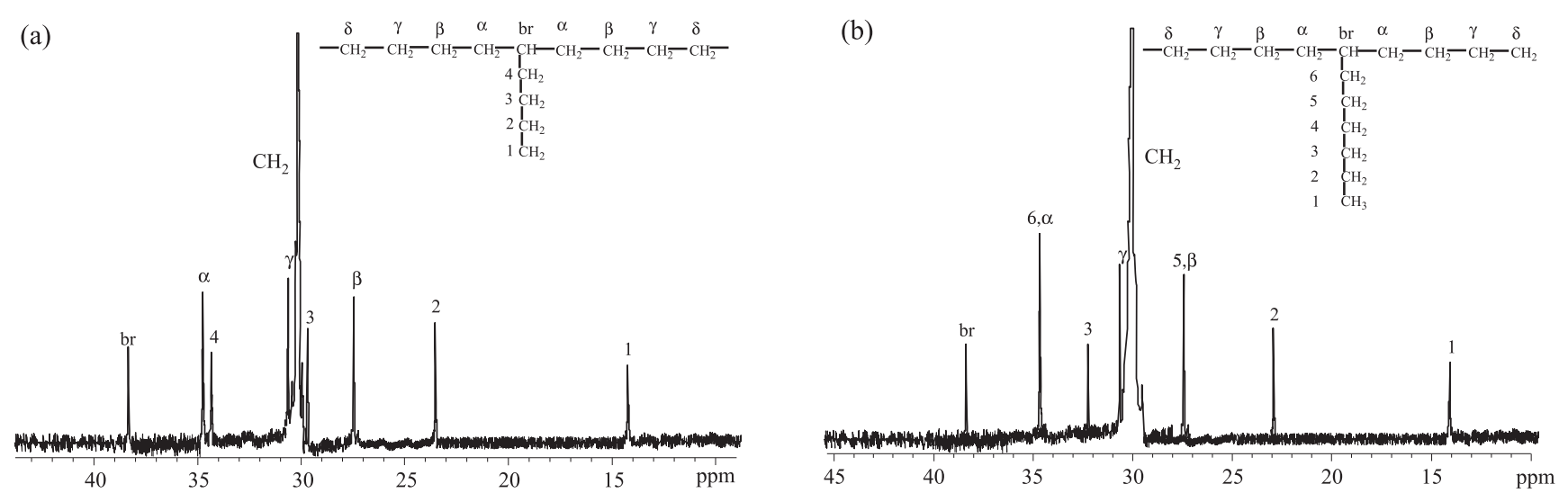

Figura 2. Espectro de RMN- ${ }^{13} \mathrm{C}$ em 1,2,4-triclorobenzeno a $125^{\circ} \mathrm{C}$. ( a) Hex-Zn; (b) Oct-ZN

carbono adjacente ao grupo metila da ramificação hexila. Como exemplo, é mostrado na Figura 1, a nomenclatura aplicada ao LLDPE com o comonômero1-octeno.

O método para a análise quantitativa utilizando o $\mathrm{RMN}^{13} \mathrm{C}$ consiste em dividir o espectro em áreas de acordo com a faixa de deslocamento químico, sendo que cada área pode conter mais de um pico. Por exemplo, a área designada pela letra $\mathrm{C}$ inclui o pico a 38,2 ppm. Nas Figuras 2(a) e 2(b) encontram-se os espectros de $\mathrm{RMN}^{13} \mathrm{C}$ para o Hex-ZN e Oct-ZN, respectivamente. Os limites de integração, os deslocamentos químicos obtidos e a atribuição de cada pico para os LLDPE estão listados na Tabela 2. É importante salientar que alguns dos deslocamentos químicos não são observados nesta análise devido à concentração dos comonômeros para os LLDPE analisados; alguns sinais só aparecem em concentrações acima de $5 \mathrm{~mol}$ $\%$, pois acima desta concentração são observadas estruturas em bloco dos comonômeros responsáveis por estes sinais, como é o caso dos picos localizados nas áreas $\mathrm{A}, \mathrm{B}$ e D, por exemplo.

Uma vez definidos os picos e obtidas as áreas via integração, aplica-se os valores às equações indicadas a seguir, utilizadas para calcular a composição em mol \%. As áreas foram obtidas por meio de ajustes de curvas proporcionando assim um

Tabela 2 - Limites de integração e dados experimentais para os LLDPE.

\begin{tabular}{|c|c|c|c|c|}
\hline Polímero & Área & $\delta(\mathbf{p p m})^{(\mathrm{a})}$ & $\delta(\mathbf{p p m})^{(\mathbf{b})}$ & Atribuição(a) \\
\hline \multirow{8}{*}{ Hexeno } & A & 41,5 a 40,5 & & Blocos $(\mathrm{C})$ \\
\hline & B & 40,5 a 39,5 & & Blocos ${ }^{(C)}$ \\
\hline & $\mathrm{C}$ & 39,5 a 37,0 & 38,2 & br \\
\hline & $\mathrm{D}$ & Pico a 35,8 & & Blocos $(\mathrm{C})$ \\
\hline & $\mathrm{D}+\mathrm{E}$ & 36,8 a 33,2 & 34,$6 ; 34,2$ & $\alpha ; 4$ \\
\hline & $F+G$ & 33,2 a 25,5 & 29,$6 ; 30,0 ; 30,5$ & $3 ; \mathrm{CH}_{2}$ isolado; $\gamma$ \\
\hline & G & 28,5 a 26,5 & 27,3 & $\beta$ \\
\hline & $\mathrm{H}$ & 24,9 a 24,1 & & \\
\hline \multirow{9}{*}{ Octeno } & A & 41,5 a 40,5 & & Blocos ${ }^{(\mathrm{C})}$ \\
\hline & B & 40,5 a 39,5 & & Blocos $(\mathrm{C})$ \\
\hline & $\mathrm{C}$ & 39,5 a 37,0 & 38,3 & br \\
\hline & $\mathrm{D}$ & Pico a 35,8 & & Blocos (C) \\
\hline & $\mathrm{D}+\mathrm{E}$ & 36,8 a 33,2 & 34,6 & $\alpha$ \\
\hline & $\mathrm{F}+\mathrm{G}+\mathrm{H}$ & 33,2 a 25,5 & 30,$0 ; 30,5 ; 32,2$ & $4 \mathrm{e} \mathrm{CH}_{2}$ isoldado; $\gamma ; 3$ \\
\hline & $\mathrm{H}$ & 28,5 a 26,5 & 27,3 & $\beta$ \\
\hline & I & 25,0 a 24,0 & & \\
\hline & $\mathrm{P}$ & 24,0 a 22,0 & 22,9 & 2 \\
\hline
\end{tabular}

(a) deslocamento químico segundo De Pooter ${ }^{[12]}$

(b) deslocamento químico obtido experimentalmente ${ }^{[1,2]}$

(c) só aparecem a concentrações superiores a $5 \mathrm{~mol} \%$ de comonômero 
cálculo mais exato.

Para o cálculo do número de moles de 1-hexeno no LLDPE Hex-ZN é necessário definir:

$\mathrm{H} 1=$ carbonos $\alpha=[1,5 \mathrm{~A}+2 \mathrm{~B}+(\mathrm{D}+\mathrm{E})-\mathrm{D}] / 3$

$\mathrm{H} 2=$ carbonos $b r=(\mathrm{A}+2 \mathrm{C}+2 \mathrm{D}) / 2$

$\mathrm{H}^{\prime}=$ média de moles de 1-hexeno $=(\mathrm{H} 1+\mathrm{H} 2) / 2$

Especificamente para o Hex-ZN deste trabalho: $A=B=D=H=$ 0 (Tabela 2)

Para o cálculo do número de moles de eteno no LLDPE Hex-ZN é necessário definir:

$E^{\prime}=[(F+G)-(3 A+3 B+G+H)] / 2+H^{\prime}$

Mol \% de 1-hexeno $=100 \%$ x H' / (E' + H')

Para o cálculo do número de moles de 1-octeno no LLDPE Oct-ZN é necessário definir:

$\mathrm{O} 1=\operatorname{carbonos} \alpha=(\mathrm{A}+2 \mathrm{C}+2 \mathrm{D}) / 2$

$\mathrm{O} 2=$ carbonos $\mathrm{br}=[1,5 \mathrm{~A}+2 \mathrm{~B}+(\mathrm{D}+\mathrm{E})-\mathrm{D}] / 3$

$\mathrm{O}^{\prime}=$ média de moles de octeno- $1=\mathrm{O} 1+\mathrm{O} 2 / 2$

Especificamente para os Oct-ZN deste trabalho: $\mathrm{A}=\mathrm{B}=\mathrm{D}=$ $\mathrm{I}=0$ (Tabela 2)

Para o cálculo do número de moles de eteno no LLDPE Oct-ZN é necessário definir:

$\mathrm{E}^{\prime}=[(\mathrm{F}+\mathrm{G}+\mathrm{H})-(3 \mathrm{~A}+3 \mathrm{~B}+\mathrm{H}+\mathrm{P}+\mathrm{I})] / 2+\mathrm{O}^{\prime}$

Mol \% de 1-octeno $=100 \%$ x O'/ $\left(\mathrm{O}^{\prime}+\mathrm{E}^{\prime}\right)$

O número de ramificações por 1000 átomos de carbono é obtida através da equação:

$\mathrm{CH}_{3} / 1000$ carbonos $=\frac{1000(\text { moles } \% \text { de comonômero }}{2(\text { moles } \% \text { de eteno })+\mathrm{n}(\text { moles } \% \text { comonômero })}$

onde $\mathrm{n}=$ número de carbonos no comonômero

Na Tabela 3 encontram-se os valores obtidos para a porcentagem em mol de comonômeros incorporados à cadeia principal, bem como o número de ramificações por 1000 átomos de carbono.

Tabela 3 - Porcentagem em mol de comonômero e número de ramificações por 1000 átomos de carbono nos LLDPE.

\begin{tabular}{cccc}
\hline Copolímero & Mol \% $^{(\mathbf{a})}$ & $\begin{array}{c}\mathbf{C H}_{\mathbf{3}} / \mathbf{1 0 0 0 C} \\
\text { (FTIR) }\end{array}$ & $\begin{array}{c}\mathbf{C H}_{3} / \mathbf{1 0 0 0 C} \\
\left(\mathbf{R M N}^{13} \mathbf{C}\right)\end{array}$ \\
\hline Hex-ZN & 3,0 & 15 & 14 \\
Oct-ZN & 2,4 & 12 & 11 \\
Oct-M $^{(b)}$ & - & 17 & 34 \\
Oct-ZN/M & 2,8 & 14 & 13 \\
\hline
\end{tabular}

(a) Determinado por $\mathrm{RMN}^{13} \mathrm{C}$

(b) Contém ramificações longas e curtas

\section{Espectroscopia no Infravermelho}

Embora a ressonância magnética nuclear de ${ }^{13} \mathrm{C}$ seja utilizada com muito freqüência, pois trata-se de um método absoluto, que permite identificar e quantificar os tipos de ramificações sem a necessidade de utilizar padrões, o tempo e o custo da análise são muito altos quando comparados com a espectroscopia no infravermelho. O uso cada vez mais freqüente da espectroscopia no infravermelho para análises de ramificações é uma conseqüência direta da sua simplicidade, rapidez e baixo custo do equipamento.

O método por FTIR utiliza a banda de absorbância do grupo metila em torno de $1377 \mathrm{~cm}^{-1}$ como referência para a determinação do teor de RCC. Este método apresenta algumas limitações como, por exemplo, a absorbância deve ser corrigida devido à interferência dos grupos metilênicos e de outros grupos. A freqüência de absorção dos grupos metilas também é, em alguns casos, dependente do tipo de ramificação e da cristalinidade, além da espessura do filme ${ }^{[16]}$.

A banda relativa a deformação da metila terminal de cada ramificação pode ser distinguida de acordo com o tipo de ramificação. Por exemplo, as ramificações etila $\left(1379 \mathrm{~cm}^{1}\right)$ e isobutila $\left(1383 \mathrm{~cm}^{1}\right)$ são facilmente distinguíveis de outras ramificações. Por outro lado, as ramificações metila, butila e hexila (todas a $1377 \mathrm{~cm}^{1}$ ) não podem ser distinguidas. Para uma análise qualitativa pode-se utilizar as bandas de absorção referentes ao grupo metila entre 1400 e $800 \mathrm{~cm}^{-1}{ }^{[17]}$.

Ao contrário da banda de deformação da metila a $1378 \mathrm{~cm}^{-1}$, que não permite diferenciar entre ramificações butila e maiores, as bandas relativas ao modo de deformação rocking do grupo metila apresentam um deslocamento de até $5 \mathrm{~cm}^{-1}$ entre as ramificações butila e hexila. As ramificações butila apresentam absorção com máximo a $894 \mathrm{~cm}^{-1}$ e as ramificações hexila a $889 \mathrm{~cm}^{-1}$. Estas duas bandas, no entanto, são parcialmente encobertas pela banda dos grupos vinila terminais a $910 \mathrm{~cm}^{-1}$, desta forma torna-se necessário uma deconvolução para a subtração desta banda ${ }^{[17]}$.

Na Figura 3 encontram-se os espectros de infravermelho

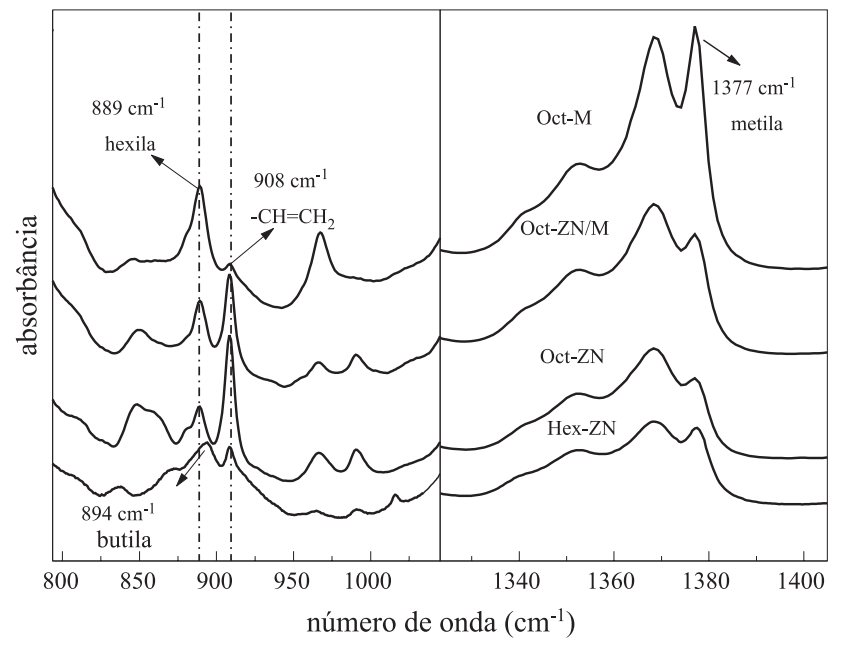

Figura 3. Espectros de FTIR - comparação das bandas de hexila, butila, vinila terminal e deformação da metila em 889, 894, 910 e $1377 \mathrm{~cm}^{-1}$, respectivamente. 
na região de 800 a $1400 \mathrm{~cm}^{-1}$ para os quatro polietilenos. Observa-se, além da banda de deformação da metila a 1377 $\mathrm{cm}^{-1}$, as bandas em torno de $889 \mathrm{~cm}^{-1}$ e $894 \mathrm{~cm}^{-1}$ relativas ao modo de deformação rocking da metila presente na ramificação hexila e butila, respectivamente, e a banda em $910 \mathrm{~cm}^{-1}$, relativa a vinila terminal. Esta banda da vinila terminal praticamente não aparece para o LLDPE Oct-M e a banda da hexila, ao contrário, é mais intensa. Além disso, a intensidade da banda em $1377 \mathrm{~cm}^{-1}$ também é mais intensa para o LLDPE Oct-M. Isso pode ser um indício de que a inserção de ramificações é maior para este LLDPE ${ }^{[1,17]}$.

Pela análise de FTIR é possível determinar o teor de ramificação por dois métodos. O método mais utilizado depende de pelo menos um padrão bem definido, que pode ser obtido por $\mathrm{RMN}^{13} \mathrm{C}$, fornecendo o teor de ramificação, número de $\mathrm{CH}_{3}$ por 1000 átomos de carbono e relacionando a intensidade da banda $1377 \mathrm{~cm}^{-1}$ com o teor de RCC. O outro método utiliza o grupo $-\mathrm{CH}_{2}-$ como padrão interno e depende da densidade do material ${ }^{[1,17,18]}$. Para utilizar o primeiro método, além da necessidade de um padrão bem definido, é preciso normalizar o espectro infravermelho utilizando-se uma segunda banda como referência a partir de uma mesma linha base (LB). Como observado na Figura 4a, o espectro do Oct$\mathrm{ZN}$ apresenta todas as bandas com menor intensidade comparativamente às demais amostras. Esta diferença na intensidade pode estar relacionada com diferenças na espessura e/ou de cristalinidade das amostras. Então tem-se, pelo

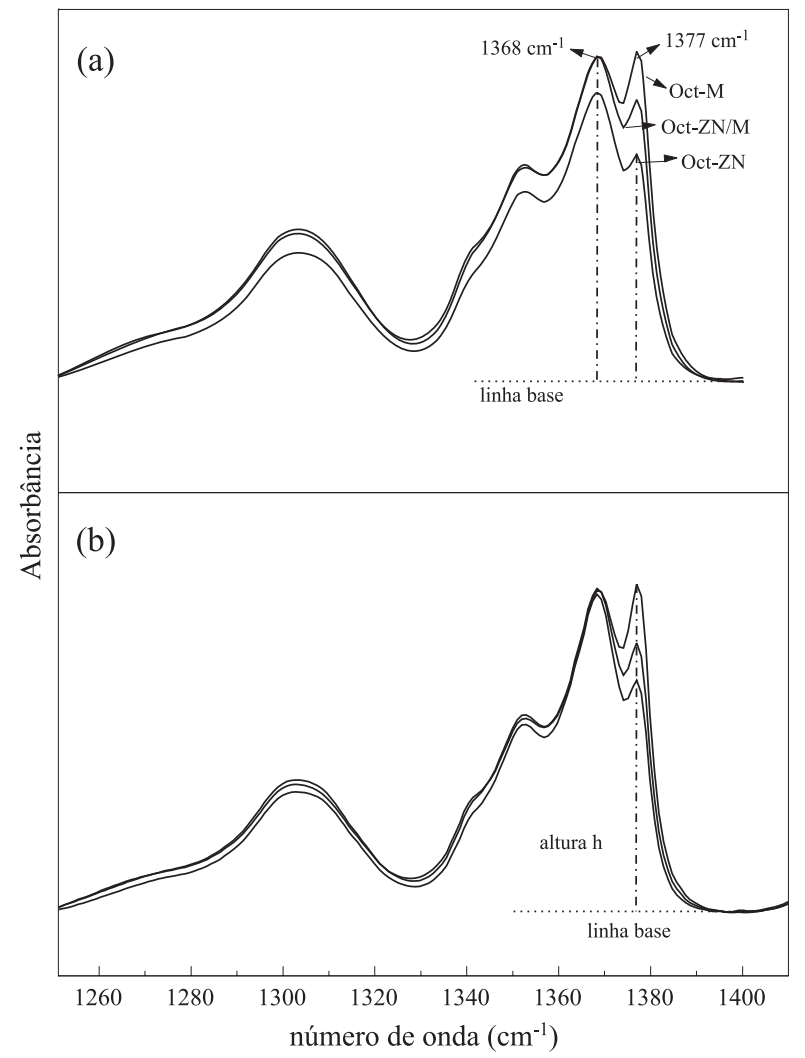

Figura 4. Espectros de FTIR dos três LLDPE com octeno. (a) espectros não normalizados e (b) espectros normalizados utilizando-se a banda a $1368 \mathrm{~cm}^{-1}$. espectro normalizado com relação a banda em $1368 \mathrm{~cm}^{-1}$ do Oct-ZN, Figura 4b:

$$
\mathrm{RCC}_{\text {amostra }}=\mathrm{RCC}_{\text {padrão }} \mathrm{X} \mathrm{h} \mathrm{h}_{\text {amostra) }} / \mathrm{h}_{\text {padrão }}
$$

Onde RCC e h são o teor de ramificações da amostra a altura da banda em $1377 \mathrm{~cm}^{-1}$, respectivamente. RCC entende-se por $\mathrm{CH} 3 / 1000 \mathrm{C}$ ou mol \%.

$\mathrm{Na}$ Tabela 3 encontram-se os valores obtidos por RMN $-{ }^{13} \mathrm{Ce}$ FTIR para o número de grupos $\mathrm{CH}_{3}$ para cada 1000 átomos de carbono. Pode-se observar que há uma concordância entre os valores de $\mathrm{CH}_{3} / 1000 \mathrm{C}$ obtidos por $\mathrm{RMN}-{ }^{13} \mathrm{C}$ e por FTIR para os LLDPE, com exceção para o LLDPE Oct-M, provavelmente devido às limitações dos dois métodos na caracterização das ramificações longas, como as presentes neste polietileno. Além disso, utilizou-se a amostra Oct-ZN como padrão para a calibração de FTIR. Em relação a amostra Oct-M é preciso considerar que na quantidade de ramificações obtida por $\mathrm{RMN}-{ }^{13} \mathrm{C}$ está embutida, indiscriminadamente, tanto RCC quanto RCL, pois a partir de 6 carbonos o método não distingue RCC de RCL e a absortividade molar da banda de deformação da metila diminui com o aumento do comprimento da ramificação, ou seja, ela varia com o tipo de ramificação. Isto pode explicar porque há discordância entre os teores de comonômeros obtidos por FTIR e por RMN- ${ }^{13} \mathrm{C}$ para o Oct-M $\mathrm{M}^{[1,2]}$.

\section{Conclusões}

A aplicação da técnica de RMN permite a determinação absoluta do teor de ramificações em polietilenos. Entretanto, requer cuidados experimentais e equipamentos trabalhando em condições diferenciadas, como alta temperatura e tempo de análise. Associado a isto, o tratamento de dados não é trivial. Já a espectroscopia infravermelho mostra-se bastante simples na obtenção e no tratamento dos dados. A desvantagem reside na necessidade de um padrão, ou seja, um polietileno com teor de ramificações bem estabelecido, cujo espectro possa ser utilizado na obtenção do teor de ramificação em análises de rotina. Os resultados obtidos por $\mathrm{RMN}^{13} \mathrm{C}$ e infravermelho para os diferentes polietilenos mostraram boa concordância entre si, exceto para o polietileno que apresenta uma combinação de cadeias curtas e longas. Neste caso, a técnica de RMN detecta todos os carbonos terciários, independentemente destes fazerem parte de uma ramificação longa ou curta. Enquanto que a técnica de espectroscopia de infravermelho é mais sensível para a quantificação de ramificações curtas.

\section{Agradecimentos}

À FAPESP processos no 98/12239-6; 98/12238-0; 99/03642-4

\section{Referências Bibliográficas}

1. Hanamoto, L.S. - "Estudo da Cinética de Cristalização de Polietileno Linear de Baixa Densidade: Influência da 
Microestrutura", Tese de Mestrado, Universidade Estadual de Campinas, UNICAMP, Brasil (2000).

2. Quental, A.C. - "Blendas de Polietileno Linear de Baixa Densidade (LLDPE) e Poli(propeno-co-eteno-co-1buteno)", Tese de Mestrado, Universidade Estadual de Campinas, UNICAMP, Brasil (2000).

3. Mark, H.F.; Bikales, N.M.; Overberger, C.G. \& Menges, G. - "Encyclopedia of Polymer Science and Engineering", vol. 6, John Wiley and Sons, New York (1986).

4. Kaminsky, W. - "Polyolefins" em "Handbook of Polymer Synthesis", Parte A, Cap.1, Hans R. Kricheldorf (ed.), Marcel Dekker Inc., New York (1992).

5. Todo, A.\& Kashiwa, N. - Macromol. Symp., 101, p.301 (1996).

6. Fu, Q.; Chiu, F.C.; McReight, K.W.; Guo, M.; Tseng, W.W. \& Cheng, S.Z.D., - J. Macromol. Sci. Phys., B 36 (1), p.41 (1997).

7. Forte, M.C.; Miranda, M.S.L. \& Dupont, J. - Polímeros: Ciência e Tecnologia, jul/set, p.49 (1996).

8. Plumley, T.A.; Sehanobish, K.; Patel, R.M.; Lai, S.Y.; Chum, S.P. \& Knight, G.W. - J. Plastic Film \& Sheeting, 12, p.269 (1996).

9. Schut, J.H. - Plastics World, sept, p.43 (1996).
10. Montagna, A.A.; Burkhart, R.M. \& Dekmezian, A.H. Chemtech, dec, p.26 (1997).

11. ASTM D5017-91 “ Standard Test Methods for Determination of Linear Low Density Polyethylene (LLDPE) Composition by Carbon-13 Nuclear Magnetic Resonance.

12. De Pooter, M.; Smith, P.B.; Dohrer, K.K.; Bennett, K.F.; Meadows, M.D.; Smith, C.G.; Schouwenaars, H.P. \& Geerards, R.A. - J. Appl. Polym. Sci., 42, p.399 (1991).

13. Hsieh, E.T. \& Randall, J.C. - Macromolecules, 15, p.1402 (1982).

14. Bugada, D.C. \& Rudin, A. - Eur. Polym. J., 23, p.809 (1987).

15. Usami, T. \& Takayama, S. - Macromolecules, 17, p. 1756 (1984).

16. ASTM 2238-92 "Standard Test Methods For Absorbance of Polyethylene Due to Methyl Groups at $1378 \mathrm{~cm}^{-1}$

17. Blitz, J. P. \& McFaddin, D.C. - J. Appl. Polym. Sci., 51, p.13 (1994).

18. Zimerman, O.E.; Cui, C.; Wang, X.; Atvars, T.D.Z. \& Weiss, R.G. - Polymer, 39, p.1177 (1998).

Enviado: $31 / 12 / 04$

Reenviado: 26/04/05

Aprovado: 09/05/05 